\title{
BMJ Open Efficacy and safety of gelatine tannate for the treatment of acute gastroenteritis in children: protocol of a randomised controlled trial
}

\author{
Dorota Michałek, Maciej Kołodziej, Zofia Konarska, Hania Szajewska
}

To cite: Michałek D, Kołodziej M, Konarska Z, et al. Efficacy and safety of gelatine tannate for the treatment of acute gastroenteritis in children: protocol of a randomised controlled trial. BMJ Open 2016;6: 010530

doi:10.1136/bmjopen-2015010530

- Prepublication history for this paper is available online. To view these files please visit the journal online (http://dx.doi.org/10.1136/ bmjopen-2015-010530).

DM and MK contributed equally.

Received 12 November 2015 Revised 22 January 2016 Accepted 25 January 2016

CrossMark

Department of Paediatrics, The Medical University of Warsaw, Warsaw, Poland

Correspondence to Professor Hania Szajewska; hania@ipgate.pl

\section{ABSTRACT}

Introduction: Worldwide, acute gastroenteritis in children, usually caused by viruses, leads to considerable morbidity and mortality. The treatment is aimed at preventing and treating dehydration, promoting weight gain after rehydration, and reducing the duration and severity of diarrhoea. Effective and inexpensive interventions that could add to the effect of oral rehydration therapy are of interest. Recently, in many European countries, gelatine tannate is being widely marketed for treating acute gastroenteritis. Gelatine tannate is a complex of tannic acid, which possesses astringent and anti-inflammatory properties, and a protective gelatine. Currently, there is no evidence to support the use of gelatine tannate for treating acute gastroenteritis in children and only scant evidence to support the use of gelatine tannate in adults. We aim to assess the efficacy of gelatine tannate for the treatment of acute gastroenteritis in children.

Methods and analysis: This will be a blind, placebocontrolled, randomised trial. Children younger than 5 years of age with acute gastroenteritis defined as a change in stool consistency to loose or liquid form (according to the Bristol Stool Form scale or Amsterdam Stool Form scale) and/or an increase in the frequency of evacuations (typically $\geq 3$ in $24 \mathrm{~h}$ ), lasting for no longer than 5 days, will be recruited. A total of 158 children will be randomised to receive either gelatine tannate (children younger than 3 years of age will receive $250 \mathrm{mg}, 4$ times/day, and those older than 3 years of age will receive $500 \mathrm{mg}, 4$ times/day) or matching placebo for 5 days. The primary outcome measure is the duration of diarrhoea.

Ethics and dissemination: The Bioethics Committee approved the study protocol. The findings of this trial will be submitted to a peer-reviewed paediatric journal. Abstracts will be submitted to relevant national and international conferences.

Trial registration number: NCT02280759;

Pre-results.

\section{INTRODUCTION}

Worldwide, acute gastroenteritis in children, usually caused by rotaviruses, leads to considerable morbidity and mortality. ${ }^{1}$ It is also the
Strengths and limitations of this study

- The study design (randomised controlled trial, RCT) is the most robust methodology to assess the effectiveness of therapeutic interventions.

- A precise clinical question has been posed to fill a gap in knowledge as to whether gelatine tannate is safe and effective in the treatment of acute gastroenteritis in children.

- The findings of this RCT, whether positive or negative, will contribute to the formulation of recommendations on the use of gelatine tannate for the treatment of acute gastroenteritis.

- Owing to practicalities, stool volume, which is one of the objective ways of assessing the efficacy of antidiarrhoeal drugs, will not be assessed.

most common cause of primary care consultations among children younger than 5 years of age. ${ }^{2}$ According to current European guidelines, ${ }^{3}$ the mainstay of treatment for acute gastroenteritis is oral rehydration with a hypoosmolar solution. Breast feeding should not be interrupted. Regular feeding should continue with no dietary changes, including milk. Considering the burden of acute gastroenteritis both to children and the healthcare system, effective and inexpensive interventions that could add to the effect of oral rehydration therapy are of interest. Currently, effective interventions that may reduce the duration and severity of diarrhoea include administration of specific probiotics such as Lactobacillus rhamnosus GG or Saccharomyces boulardii, diosmectite or racecadotril. ${ }^{3}$

Recently, in many European countries, gelatine tannate is being marketed for treating acute gastroenteritis. Gelatine tannate consists of tannic acid suspended in a gelatine solution. Gelatine tannate has a stable structure both in the acidic environment of the stomach as well as in a basic and neutral environment such as in the small intestine and colon. ${ }^{4}$ Little is known about the specific 
mechanisms by which gelatine tannate may act against gastrointestinal infection. It is known, however, that it forms a biofilm, which mechanically protects the gastrointestinal mucosa and causes precipitation of proinflammatory proteins such as mucoproteins in the intestinal mucosa. ${ }^{5}$ In addition, it inhibits the growth of bacteria such as Bacteroides fragilis, Clostridium perfringens, Escherichia coli, Enterobacter cloacae, Salmonella typhimurium, Helicobacter pylori, Listeria monocytogenes, and in vitro mycobacterial Vibrio cholerae. ${ }^{5-7}$ The action of anti-inflammatory tannate also involves blocking inflammatory agents in the gastrointestinal mucosa. ${ }^{8}$

Only limited evidence is available on the effectiveness of gelatine tannate. In 2009, Esteban Carretero et al published a study ( $\mathrm{n}=211$, mean age $2.5 \pm 2.4$ years, no randomisation, no blinding, unbalanced baseline characteristics) that evaluated the effectiveness of gelatine tannate in the treatment of acute gastroenteritis in children. Children received oral rehydration solution (ORS) alone or ORS in combination with gelatine tannate (dose was not specified). During the $12 \mathrm{~h}$ observation period, a significant reduction in the number of loose stools was found in the group receiving ORS and gelatine tannate versus the group receiving ORS alone. Both groups had similar weight gains, stool consistency, comparative risks of diarrhoea with blood, peritonitis, and sepsis, vomiting intensity, and degrees of dehydration in the $12 \mathrm{~h}$ of observation. ${ }^{9}$ In 2012, Allegrini and Costantini performed a blinded, randomised, placebocontrolled trial in the adult population $(n=40$, mean age $43 \pm 13$ years). In the group receiving gelatine tannate (500 mg, 6 times/day, for 2 consecutive days) compared with the placebo group, a statistically significant decrease in both the daily number of watery stools and severity of abdominal pain for the first $48 \mathrm{~h}$ of the intervention was found. There were no clinically relevant adverse events. ${ }^{10}$

A 2014 systematic review evaluated the efficacy of gelatine tannate in treating acute gastroenteritis in children and adults. ${ }^{11}$ The two studies aforementioned were included. None of the included studies evaluated the effect of gelatine tannate on the primary outcome measures for the review such as stool output, duration of diarrhoea, need for admission to the hospital, duration of hospital stay, and (in children) weight gain after rehydration. The review concluded that there is no evidence to support the use of gelatine tannate for treating acute gastroenteritis in children and only sparse evidence to support the use of gelatine tannate in adults.

Currently, the total evidence to support the use of gelatine tannate for treating acute gastroenteritis in children or adults is very limited. According to the current (2014) European guidelines, ${ }^{3}$ gelatine tannate is not recommended for the management of acute gastroenteritis in children.

\section{Trial objectives and hypotheses}

The main objective of this trial is to assess the effectiveness and safety of gelatine tannate in the treatment of acute gastroenteritis in children. We aim to conduct a well-designed study, with sufficient power, an adequate follow-up period, and relevant clinical end points. In our trial, we choose to use placebo for a comparator as it is widely regarded as the gold standard for testing the efficacy of new treatments. ${ }^{12}$

\section{METHODS AND ANALYSIS}

The trial is registered at http://www.clinicaltrials.gov (NCT02280759), and any important changes in the protocol will be implemented there.

\section{Study design}

This study is designed as a randomised, blinded, placebo-controlled trial, with allocation $1: 1$, and is described in more detail in the subsequent sections.

\section{Setting and participants}

The recruitment will take place primarily in the emergency room of the paediatric hospital of the Medical University of Warsaw. However, other recruiting sites are under consideration provided that the personnel are adequately trained and competent in conducting clinical trials. Participants will be randomised after their first visit to the emergency room or after admission to the clinic. Caregivers will receive oral and written information on the study. Written informed consent will by obtained by physicians involved in the study.

\section{Inclusion criteria}

Children eligible for the trial must fulfil all of the following criteria:

- Acute gastroenteritis defined as a change in stool consistency to loose or liquid form (according to the Bristol Stool Form (BSF) scale or in the case of infants, the Amsterdam Stool Form (ASF) scale) and/ or an increase in the frequency of evacuations (typically $\geq 3$ in $24 \mathrm{~h}$ ), lasting for no longer than 5 days;

- Age younger than 5 years;

- A caregiver must provide written informed consent.

\section{Exclusion criteria}

- Use of antibiotics, gelatine tannate, diosmectite, probiotics, racecadotril, or zinc (including zinc containing ORS) within a week prior to enrolment

- Exclusive breast feeding

- Chronic diarrhoeal gastrointestinal disease (eg, inflammatory bowel diseases, cystic fibrosis, coeliac disease, food allergy)

- Immunodeficiencies

- Malnutrition (weight/height/length under 3rd percentile) (WHO Child Growth Standards will be used) ${ }^{13}$

- If needed, discontinuation or modification of the treatment may be considered at the discretion of the physician.

\section{Randomisation criteria}

After rechecking the inclusion and exclusion criteria, participants will be assigned into one of two groups 
(experimental or control). After determination of eligibility, caregivers will receive a diary of symptoms to record the number of stools and stool consistency during the intervention (including recording of the timing of stools); BSF and ASF scales will be provided. Additionally, caregivers will be asked to write down any adverse events during the intervention period.

\section{Interventions}

The intervention under investigation is gelatine tannate manufactured by ICN Polfa Rzeszów/Valeant. The manufacturer does not have and will not have a role in the design or conduct of the study. The placebo will contain maltodextrin, which is an almost flavourless, easily digestible polysaccharide commonly used as a food additive. The dose of the active product or placebo will be age dependent (ie, in children younger than 3 years of age, the dose is $250 \mathrm{mg}$, and in children older than 3 year of age$500 \mathrm{mg})$. Both the gelatine tannate and placebo will be taken orally, 4 times/day, for 5 days. Caregivers will be instructed to administer the daily dose at the same time in a day after mixing the contents of the sachet with a small amount of water. The study products used in this trial will be prepared by the hospital pharmacy at the Medical University of Warsaw as sachets that seem to be identical. Table 1 for timetable of activities planned during the study.

For initial rehydration, all children will be treated according to 2014 European recommendations (fast oral rehydration over $3-4 \mathrm{~h}$ by mouth or via nasogastric tube with the recommended hypotonic solution). After all the signs of dehydration have disappeared, ORS will be given for ongoing losses until the diarrhoea stops. Rapid reintroduction of the previous diet after successful rehydration will be recommended. At all times, breast feeding will be allowed.

At any time, caregivers will have the right to withdraw the participating child from the study; they will be not obliged to give reasons for this decision, and there will be no effect on subsequent physician and/or institutional medical care.

\section{Concomitant medications}

The concomitant administration of any other medication, including antipyretics and antiemetics, will be at the discretion of the physician to provide adequate care. However, it is recommended that no unnecessary

\begin{tabular}{|c|c|c|c|c|c|c|c|}
\hline \multirow[b]{2}{*}{ Activity } & \multicolumn{7}{|c|}{ Days } \\
\hline & 1 & 2 & 3 & 4 & 5 & 6 & 7 \\
\hline Enrolment & + & & & & & & \\
\hline Randomisation & + & & & & & & \\
\hline Intervention & + & + & + & + & + & & \\
\hline Return of diary & & & & & + & & \\
\hline Follow-up & & & & & & + & + \\
\hline Adverse events & + & + & + & + & + & + & + \\
\hline
\end{tabular}

concomitant medication be used. In particular, the use of antibiotics, diosmectite, probiotics, or racecadotril (all included in the exclusion criteria) should be avoided.

\section{Follow-up}

All study participants will be followed up for the duration of the intervention ( 5 days), and then for an additional $48 \mathrm{~h}$.

\section{Allocation concealment and blinding}

A computer-generated randomisation list prepared by a person unrelated to the trial will be used to allocate participants to the study groups in blocks of eight. Consecutive randomisation numbers will be given to participants at enrolment. This procedure will be performed by one of the two physicians involved. The study product will be weighed, packaged, and signed by consecutive numbers according to the randomisation list of the hospital pharmacy at the Medical University of Warsaw by independent personnel not involved in the conduct of the study. The study products will be delivered to the physicians in small envelopes labelled with the consecutive numbers and dose (with the meaning of numbers blinded and information deposited in a sealed envelope in a safe place in the administrative part of the department). The active product and placebo will be packaged in identical sachets. Contents will look and taste the same. Researchers, caregivers, outcome assessors, and a person responsible for the statistical analysis will be blinded to the intervention until the completion of the study. The information on intervention assignments will be stored in a sealed envelope in a safe place in the administrative part of the department. The personal information about potential and enrolled participants will be stored in a locker within the study site, accessible for the involved researchers only.

\section{Compliance}

The caregivers will be asked to bring the remaining study product and diary to the study site at the end of the intervention period. Compliance with the study protocol will be checked by counting the number of sachets left unused. Based on previously published trials, it seems to be appropriate to consider those participants receiving $<75 \%$ of the recommended doses as noncompliant.

\section{Primary outcome}

The primary outcome will be the duration of diarrhoea, defined as the time until the normalisation of stool consistency according to the BSF or ASF scale (in BSF scale, numbers 2, 3, 4 and 5; in ASF scale, letters B or C), or the time until the normalisation of the number of stools (compared with the period before the onset of diarrhoea), and the presence of normal stools for $48 \mathrm{~h}$.

\section{Secondary outcomes}

- Need for intravenous rehydration

- Need for hospitalisation of outpatients 
- Number of watery stools per day

- Vomiting

Weight gain

- Adverse events

- Recurrence of diarrhoea (48 h after intervention)

- Severity of diarrhoea according to Vesikari scale ${ }^{14}$

- Use of concomitant medications

\section{Power calculation}

The primary outcome of the study is the duration of diarrhoea. Based on available data in the literature, the average duration of gastroenteritis in children is $5-7$ days. ${ }^{3}$ We assume that a clinically significant difference in the effectiveness of gelatine tannate versus placebo will shorten the duration of symptoms by 24 hours. To detect such a difference in the duration of diarrhoea between the study groups with a power of $90 \%$ and $\alpha=0.01$, a sample of 60 children is needed. Assuming approximately $20 \%$ loss to follow-up, we aim to recruit a total of 72 children for this study. In the Department of Paediatrics of the Medical University of Warsaw, there are 200 admissions of children with diarrhoea per year, and the same number of such patients who present to the emergency room. Assuming that $20 \%$ of these children will be eligible for the study, we will achieve adequate participant enrolment to reach the target sample size after 2 years of recruiting.

\section{Statistical analysis}

All analysis will be conducted on an intention-to-treat basis, including all patients in the groups to which they are randomised for whom outcomes will be available (including drop-outs and withdrawals). Descriptive statistics will be used to summarise baseline characteristics. The Student t test will be used to compare mean values of continuous variables for approximating a normal distribution. For non-normally distributed variables, the Mann-Whitney $U$ test will be used. The $\chi^{2}$ test or Fisher exact test will be used, when appropriate, to compare percentages. For continuous outcomes, differences in means or differences in medians (depending on the distribution of the data), and for dichotomous outcomes, the relative risk (RR) and number needed to treat, all with a $95 \%$ CI, will be calculated. The difference between study groups will be considered significant when the $\mathrm{p}$ value is $<0.05$, when the $95 \%$ CI for RR does not include 1.0 , or when the $95 \%$ CI for mean difference (MD) does not include 0 . All statistical tests will be two tailed and performed at the $5 \%$ level of significance.

\section{ETHICS AND DISSEMINATION}

The Bioethical Committee of The Medical University of Warsaw issued approval for the study before recruitment commenced. Verbal and written information regarding informed consent will be presented to the caregivers. Any modifications to the protocol that may affect the conduct of the study will be presented to the Bioethical
Committee. The full protocol will be available freely due to open-access publication. The findings of this randomised controlled trial will be submitted to a peerreviewed journal. Abstracts will be submitted to relevant national and international conferences.

Contributors HS conceptualised the study. All the authors contributed to the design of the study. DM and MK developed the first draft of the manuscript and contributed equally. All the authors contributed to the development of the study protocol and approved the final draft of the manuscript.

Funding This study will be fully funded by the Medical University of Warsaw. Competing interests None declared.

Ethics approval The Bioethics Committee of the Medical University of Warsaw.

Provenance and peer review Not commissioned; externally peer reviewed.

Open Access This is an Open Access article distributed in accordance with the Creative Commons Attribution Non Commercial (CC BY-NC 4.0) license, which permits others to distribute, remix, adapt, build upon this work noncommercially, and license their derivative works on different terms, provided the original work is properly cited and the use is non-commercial. See: http:// creativecommons.org/licenses/by-nc/4.0/

\section{REFERENCES}

1. Ogilvie I, Khoury $\mathrm{H}$, Goetghebeur MM, et al. Burden of community-acquired and nosocomial rotavirus gastroenteritis in the pediatric population of Western Europe: a scoping review. BMC Infect Dis 2012;12:62.

2. Van Damme $P$, Giaquinto $C$, Huet F, et al. Multicenter prospective study of the burden of rotavirus acute gastroenteritis in Europe, 2004-2005: the REVEAL study. J Infect Dis 2007;195(Suppl 1): S4-16.

3. Guarino A, Ashkenazi S, Gendrel D, et al. European Society for Pediatric Gastroenterology, Hepatology, and Nutrition/European Society for Pediatric Infectious Diseases evidence-based guidelines for the management of acute gastroenteritis in children in Europe: update 2014. J Pediatr Gastroenterol Nutr 2014;59:132-52.

4. Bueno L, Sekkal S, Theodoru V, et al. Undissociated gelatine tannate reduces intestinal leakiness and mucosa inflammation by forming a protective biofilm: results from in-vitro and in-vivo studies. United European. Gastroenterol J 2013;l:A75-6.

5. Chung KT, Stevens SE Jr, Lin WF, et al. Growth inhibition of selected food-borne bacteria by tannic acid, propyl gallate and related compounds. Lett Appl Microbiol 1993;17:29-32.

6. Scalbert A. Antimicrobial properties of tannins. Phytochemistry 1991;30:3875-83.

7. Oi $\mathrm{H}$, Matsuura $\mathrm{D}$, Miyake $\mathrm{M}$, et al. Identification in traditional herbal medications and confirmation by synthesis of factors that inhibit cholera toxin-induced fluid accumulation. Proc Natl Acad Sci USA 2002;99:3042-6.

8. Frasca G, Cardile V, Puglia C, et al. Gelatine tannate reduces the proinflammatory effects of lipopolysaccharide in human intestinal epithelial cells. Clin Exp Gastroenterol 2012;5:61-7.

9. Esteban Carretero J, Durbán Reguera F, López-Argüeta Alvarez S, et al. A comparative analysis of response to ORS (oral rehydration solution) vs. ORS+gelatin tannate in two cohorts of pediatric patients with acute diarrhea. Rev Esp Enferm Dig 2009;101:41-8.

10. Allegrini A, Costantini M. Gelatine tannate for the treatment of acute diarrhoea in adults. J Gastroint Dig Syst 2012:2;3.

11. Ruszczynski M, Urbanska M, Szajewska H. Gelatin tannate for treating acute gastroenteritis: a systematic review. Ann Gastroenterol 2014;27:121-4.

12. Benson $\mathrm{H}$, Friedman R. Harnessing the power of the placebo effect and renaming it "remembered wellness". Annu Rev Med 1996;47:193-9.

13. The WHO Child Growth Standards. http://www.who.int/childgrowth/ en/

14. Schnadower D, Tarr PI, Gorelick $\mathrm{MH}$, et al. Validation of the modified Vesikari score in children with gastroenteritis in 5 US emergency departments. J Pediatr Gastroenterol Nutr 2013;57:514-19. 
Correction: Efficacy and safety of gelatine tannate for the treatment of acute gastroenteritis in children: protocol of a randomised controlled trial

Michałek D, Kołodziej M, Konarska Z, et al. Efficacy and safety of gelatine tannate for the treatment of acute gastroenteritis in children: protocol of a randomised controlled trial. BMJ Open 2016;6:e010530. doi: 10.1136/bmjopen-2015-010530

In the Abstract, the number of children to be recruited should be 72 (as shown under Power calculation).

Open Access This is an Open Access article distributed in accordance with the Creative Commons Attribution Non Commercial (CC BY-NC 4.0) license, which permits others to distribute, remix, adapt, build upon this work non-commercially, and license their derivative works on different terms, provided the original work is properly cited and the use is non-commercial. See: http://creativecommons.org/licenses/by-nc/4.0/

C Article author(s) (or their employer(s) unless otherwise stated in the text of the article) 2017. All rights reserved. No commercial use is permitted unless otherwise expressly granted.

BMJ Open 2017;0:e010530corr1. doi:10.1136/bmjopen-2015-010530corr1

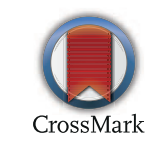

